\title{
A TECHNIQUE FOR THE CONTROLLED GROWTH OF EXCISED PLANT TISSUE IN LIQUID MEDIA UNDER ASEPTIC CONDITIONS
}

\author{
By Dr. S. M. CAPLIN* and Prof. F. C. STEWARD \\ Department of Botany, University of Rochester, New York
}

$\mathrm{E}^{\mathrm{x}}$ XCISED plant tissues are commonly grown on semi-solid father than liquid media. Immersion of the cultures in liquid media usually reduces the rate of growth (a result presumably of reduced oxygen supply), while an efiect on the ability of a culture to organise has been reported ${ }^{1}$. Given an inherent capacity of a tissue to grow, it is apparent that the growth-rate on an agar medium may well befimited by the rate of diffusion of nutrients through the agar. Hence the growth of such cultures may be more rapid on a liquid medium which is continually mixed, provided that access to oxygen is adequate. Growth in liquid media has obvious advantages wherever quantitative experiments on the absorption or nutritive effect of specific solutes is in question. It will be shown that for certain types of culture the liquid medium produces greater and more uniform growth.

DeRopp ${ }^{2}$ has described an apparatus using liquid media for growth of excised plant tissues. In this technique the cultures were to be alternately bathed in the nutrient medium, then drained and exposed to air ; but no growth-data from the use of this apparatus have been reported. Moreover, the technique appears rather unwieldy and hence is adaptable only to a relatively small number of cultures.

In the University of Rochester other methods have been devised. The method reported here has been found to be most satisfactory and instructive for the growth of excised plant tissue cultures, and is now described because it may be useful to other workers and may find further application in other biological fields.

In brief, the method makes use of a new type of culture tube: the tubes are mounted on disks which are slowly revolved about a horizontal shaft so that each tube is tumbled end over end.

The culture tube (Fig. $1 A$ ) is made from 'Pyrex' glass tubing sealed and rounded at each end. At the

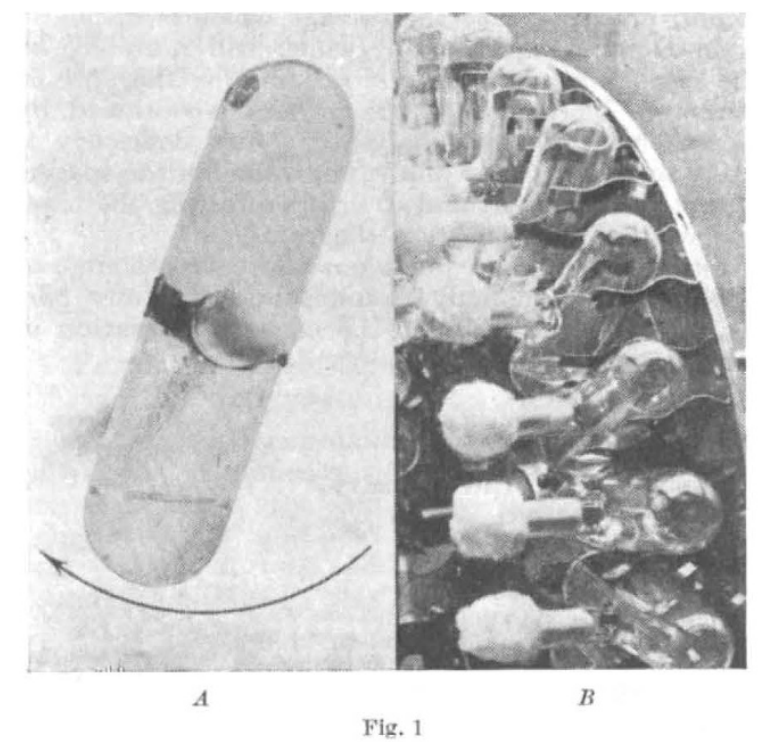

middle of each tube is a side neck through which the transfer of cultures can be made and, when plugged with cotton, through which gas exchange can occur. The culture tubes are mounted near the periphery of a circular disk of plywood so that the long axis of each tube lies along a radius of the disk and the side neck is parallel to the shaft (Fig. $1 B$ ). Twenty-four tubes $(35 \mathrm{~mm}$. in diameter and $125 \mathrm{~mm}$. long) can be fastened to each disk, and many disks may in turn be fastened to a single shaft: for example, eight disks, accommodating 192 tubes, can be mounted on a shaft $40 \mathrm{in}$. long. The shaft is driven by a motor and is geared to 1 r.p.m. In this Department the entire shaft is mounted in a cabinet in which constant temperature, humidity and light may be maintained. The revolving klinostat equalizes gravitational stimuli and ensures that all the cultures are subjected to the same environmental conditions.

As a tube 'tumbles' on the rotating klinostat the wet piece of tissue adheres at one end of the tube (Fig. $1 A$ ), whereas the liquid moves slowly from one end of the tube to the other, and as it does so, gaseous exchange with the outside air is maintained. It will readily be appreciated that with a constant volume of nutrient the fraction of the total time in which the tissue is immersed varies with the proportions of the tube; also with a tube of fixed dimensions the period in air decreases as the volume of nutrient is increased. For any given tube an upper limit to the volume of solution is set when the liquid, flowing along the now horizontal tube, tends to wet the plug. Somewhat larger volumes may be used if the axis of the klinostat is inclined to the horizontal. With these factors in mind, the dimensions of the tube have been fixed as indicated above. Ten ml. of a nutrient medium in a tube is convenient when maximal time in air is desired and also when such factors as the length of growing period for which nutrient must be furnished are taken into account.

In a previous note ${ }^{3}$ it was shown that uniform 4-mgm. cylinders cut from carrot root will grow at rates far exceeding those previously described if coconut milk is added to the nutrient agar medium. In the present experiments carrot cultures were grown both in test tubes on agar and in liquid medium on the revolving klinostat described. All the cultures were exposed to the same external conditions and were exposed to the continuous light of a bank of fluorescent lamps. The cultures in the klinostat tubes were grown on a basal nutrient medium ${ }^{4}$ to which was added 15 per cent by volume of coconut milk. For the cultures grown in test-tubes 0.8 per cent agar was added to the nutrient medium. All the explants were obtained from carrot cylinders, removed by a cannula, and cut with a multibladed cutter into 4-mgm. radial cylinders. Only phlom cylinders with their inner tangential surface $1 \mathrm{~mm}$. from the cambium were used. This technique and its implications will be described elsewhere.

* Postdoctoral Research Fellow, National Institute of Health, Washington, D.C. 


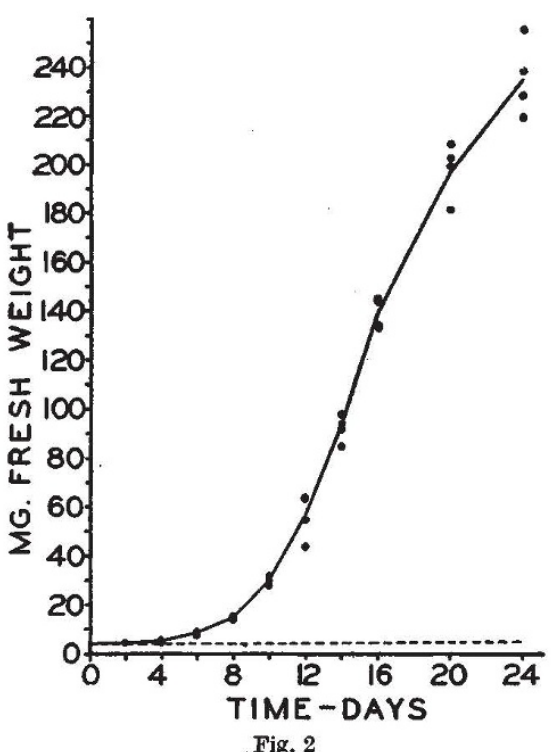

In a given experiment, cultures grown in the klinostat tubes were not only larger and more uniform but also, under the standardized conditions of the klinostat, the growth-rates of carrot cultures are much more reproducible than when the cultures are grown in nutrient agar in the test tubes.

Fig. 2 shows a growth-curve of cultures of carrot initially $4 \mathrm{mgm}$. in weight. At each period four replicate cultures were weighed, and the curve is compiled from their means. There is evidently a lag of about four days followed by a very rapid growth reaching a compound-interest rate of increase in twelve days of $\mathbf{3 4 . 1}$ per cent per day. After some days a gradual decrease in the growth-rate occurs. This decrease is probably due in part to depletion of nutrients in the culture medium. (Changing the culture solution after some fourteen days does increase the growth.)

Single cultures readily grow from a 4-mgm. explant to about $175 \mathrm{mgm}$. before they become so large that they do not readily adhere to the glass, and so fall as the tube rotates. The amount of tissue grown in a given tube and medium may be increased by using more explants per tube, but this markedly reduces the size of each cultured explant. For the most part, the technique has been applied to cultures freshly excised from the carrot root. The rates of growth of subcultures tend to be more variable, probably because they cannot readily be cut into pieces of uniform size and shape which contain identical pro. portions of active cells.

Cultures grown in liquid media in klinostat tubes and on agar differ in form: the former become compact spheroids, especially when grown in the light, while those on agar become much more succulent and irregularly concave to the medium. In the dark, cultures in klinostat tubes become friable and tend to fall apart. These differences are being investigated and will be reported elsewhere. In the dark, carrot phlœm cultures change from deep orange to pale. In the light they become pale green. Cultures from certain carrots in the light have, however, been observed to become unpredictably coloured with a red pigment in the surface layers of cells. This does not, however, affect the conclusions on the efficacy of the technique here described.
Clearly this technique is applicable to the growth of other materials in liquid media. The growth of Chlorella vulgaris in the klinostat tubes was found to compare very favourably with growth under other conditions in liquid media as used by other workers. Ten $\mathrm{ml}$. of the basal culture solution, in equilibrium with the carbon dioxide of the air, inoculated to contain 75 cells per $\mathrm{mm}^{3}$, produced in 7 days at $25^{\circ}$ C. 57,000 cells per mm. ${ }^{3}$ at a light intensity of 240 foot-candles. The technique may well find application in the experimental growth of fungi for antibiotic studies, growth studies of yeast, etc. It has, however, proved its use in quantitative investigations on the growth of plant tissue cultures.

${ }^{1}$ White, P. R., Bull. Torrey Bot. Club., 66, 507 (1939).

${ }^{2}$ DeRopp, R. S., Science, 104, 371 (1946).

${ }^{3}$ Caplin, S. M., and Steward, F. C., Science. 108, 655 (Dec. 1948).

"White, P. R., "A Handbook of Plant Tissue Culture" (Jaques Cattell Press, Lancaster, Pa.).

\section{EQUILIBRIUM BETWEEN METHYL- ACENES AND METHYLENE- DIHYDROACENES}

\author{
By DR. E. CLAR* and J. W. WRIGHT \\ Chemistry Department, University of Glaszow
}

TAUTOMERISM between methyl-acenes (I, II, III, IV, V) and methylene-dihydroacenes (Ia, II $a$, III $a$, IV $a, \mathrm{~V} a$, respectively) has often been postulated to explain side-chain reactivity of aromatic compounds, but hitherto no methylene-dihydroacene has been isolated. This weakness in the hypothesis has now been removed by the synthesis of pure 6-methylene-6 : 13-dihydropentacene (Va). The 'anellation principle'1 requires a gradual transition in properties in the acene series, and it may be inferred, therefore, that (IV), (III), (II) and (I) will also contain the corresponding methylene forms, but in decreasing proportions.<smiles>C=C1C=CCC1=Cc1ccc(C)cc1</smiles><smiles>C=C1C=CCc2ccccc21</smiles><smiles>Cc1c2ccccc2cc2ccccc12</smiles>

1<smiles>C=C1c2ccccc2Cc2ccccc21</smiles>

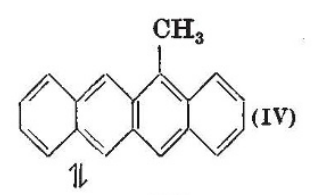<smiles></smiles><smiles>C=C1c2ccccc2Cc2cc3ccccc3cc21</smiles><smiles>C=C1c2cc3ccccc3cc2Cc2cc3ccccc3cc21</smiles>

* I.C.I. Research Fellow. 